\title{
Fabrication of a high-density emitter array for electrospray thrusters using field emitter array process
}

Naoki Inoue ${ }^{1,2^{*}}$, Masayoshi Nagao ${ }^{2}$, Katsuhisa Murakami ${ }^{2}$, Sommawan Khumpuang ${ }^{2,3}$,

Shiro Hara ${ }^{2,3}$ and Yoshinori Takao ${ }^{4 *}$

${ }^{1}$ Department of Systems Integration, Yokohama National University, Yokohama, Kanagawa 240-8501, Japan

${ }^{2}$ National Institute of Advanced Industrial Science and Technology, Tsukuba, Ibaraki 305-8568, Japan

${ }^{3}$ Minimal Fab Promoting Organization, Tsukuba, Ibaraki 305-8568, Japan

${ }^{4}$ Division of Systems Research, Yokohama National University, Yokohama, Kanagawa 2408501, Japan

*E-mail: inoe-naoki-vc@ynu.jp; takao-yoshinori-yk@ynu.jp

\section{Abstract}

To improve the thrust density of electrospray thrusters using ionic liquids as the propellant, we have fabricated a high-density emitter array utilizing the fabrication technique of a field emitter array (FEA) or gated nano electron sources. The density of the emitter array can reach approximately 4 million $/ \mathrm{cm}^{2}$, which is four orders of magnitude higher than that of conventional electrospray thrusters. Performing deep reactive ion etching from the back side of the same 
structure as FEAs and removing their Ni cones by dilute nitric acid, we have successfully fabricated sub- $\mu \mathrm{m}$ scale capillary emitters with self-aligned extractor electrodes and ionic liquid reservoirs behind them. Current measurements were also conducted with EMI-BF4 and EMIDCA as the propellant. Although the ion was not extracted with EMI-BF4 owing to its electrochemical reaction with the electrodes, both positive and negative ion currents were detected using EMI-DCA, where the current density exceeded that of conventional electrospray thrusters. 


\section{Introduction}

Recently, nano/microsatellites $(1-50 \mathrm{~kg})$ have been attracting much attention in the field of space development because they can be developed at lower cost and in a shorter term; ${ }^{1)}$ not only large companies and government agencies ${ }^{2,3)}$ but also small venture companies and universities have entered the development of nano/microsatellites. ${ }^{4-9)}$ The number of nano/microsatellites launched was more than 300 in 2017 , and it is expected to continue to increase in the future. ${ }^{10)}$ Moreover, there have been many plans to utilize nano/microsatellites in advanced missions such as constellation and deep-space exploration, ${ }^{8,9,11)}$ where high-performance small thrusters are required to do orbit transition by themselves. ${ }^{12)}$

One of the candidates for such thrusters is ion thrusters, which generally employ xenon gas as a propellant and produce thrust by emitting and accelerating ions from plasma sources together with electrons from neutralizers to keep charge neutrality. ${ }^{13)}$ Ion thrusters were already mounted on two $50 \mathrm{~kg}$-class microsatellites and successfully operated in space. ${ }^{7,8)}$ However, further miniaturization of ion thrusters is difficult because of a massive and bulky propellant feed system consisting of a high-pressure gas tank, regulator, and so on. Hence, it is still unrealistic to install ion thrusters on $10 \mathrm{~kg}$-class nanosatellites unless the high-pressure gas feeding system is removed.

To overcome this issue and to realize high-performance microthrusters that can be mounted on $10 \mathrm{~kg}$-class or even smaller nanosatellites, electrospray thrusters using ionic liquids as a propellant are one of the solutions because they do not require a gas propellant and a 
neutralizer as described below, which can drastically reduce the entire propulsion system. Figure 1 shows a schematic of an internally fed type electrospray thruster. ${ }^{14)}$ By applying a high voltage to the emitter substrate, the strong electric field is generated at the emitter tips, which pulls and deforms the ionic liquid. When the force of the field becomes stronger than the surface tension pressure of the ionic liquid, it forms a conic shape called Taylor cone. ${ }^{15)}$ At the same time, ions are emitted from the tip of the Taylor cone and accelerated by the potential difference between the emitter and the extractor, which delivers the thrust.

Ionic liquids consist of only positive and negative ions (cations and anions), and their vapor pressure is almost zero because of the strong Coulomb force between both ions, ${ }^{16)}$ indicating that ionic liquids can exist as a liquid phase even in vacuum. Moreover, since electrospray thrusters can extract and accelerate both cations and anions by applying bipolar pulse voltage, neutralizers are not required. It should be noted that the molecular weight of anions is similar to that of cations and thus anions can also contribute to the thrust, unlike light electrons of neutralizers.

Many studies of electrospray thrusters have been conducted already, ${ }^{14,17-21)}$ but obtainable thrust density is still too low compared with conventional ion thrusters, which have relatively low thrust density among electric propulsion systems. Since the obtainable thrust per single emitter is very tiny (on the order of $10 \mathrm{nN}$ ), the emitter substrate should consist of many emitters. The emitter array is usually fabricated by a top-down process with a MEMS etching technology, and the size and interval of emitters are on the order of several hundred 
micrometers; thus, up to several hundreds of emitters can be packaged per square centimeter, which is still too few to realize high thrust density. ${ }^{17)}$ For further high-density packaging, it is necessary to fabricate much smaller emitters. However, they are difficult to fabricate by the conventional top-down process together with a wafer-level bonding of the extractor electrode with sub- $\mu \mathrm{m}$ scale precision, and thus a new fabrication technique is required. In addition to the low-density issue, emission of droplets is also a problem for electrospray thrusters because it leads to low specific impulse (i.e., low fuel efficiency) ${ }^{14)}$ This problem could also be resolved by further miniaturization of emitters as will be described later in Sect. 2 .

In this study, we have proposed a new fabrication method of a high-density emitter array employing a fabrication technique of field emitter arrays (FEAs) or gated nano electron sources, ${ }^{22,23)}$ where the emitter density can be four orders of magnitude higher than that of conventional electrospray thrusters. The fabrication technology of FEAs is based on a bottomup process rather than a top-down process, and thus sub- $\mu \mathrm{m}$ scale capillary emitters with selfaligned extractor electrodes can be realized. The objective of this study is to demonstrate the fabrication of the high-density emitter array and the ion emission from such tiny capillary emitters. In Sect. 2, we propose a design concept of the high-density emitter array, followed by the description of its fabrication process together with the demonstration of the emitter array in Sect. 3. As a preliminary experiment of ion extraction, the result of current measurements is then given and discussed in Sect. 4, where the ion emission from the sub- $\mu \mathrm{m}$ scale capillaries of the high-density emitter array was detected. Finally, conclusions are presented in Sect. 5 . 


\section{Design concept of a high-density emitter array}

Figure 2 shows a conceptual design of an electrospray thruster, which consists of emitter, extractor, and accelerator electrodes for acceleration of ion beams extracted from ionic liquid to obtain high thrust density. The propellant of the ionic liquid is fed from the back side of the emitter array and through the capillary emitters. The main feature of the present electrospray thruster is a high density of the emitter array, where a single capillary emitter has a sub- $\mu \mathrm{m}$ aperture and the pitch of the capillary emitters is set at $5 \mu \mathrm{m}$. Thus, the density of the emitter array can reach approximately 4 million $/ \mathrm{cm}^{2}$, which is four orders of magnitude higher than that of conventional electrospray thrusters. ${ }^{17,21)}$ For a constraint of the fabrication (detailed in Sect. 3), the scale of an ionic liquid reservoir is set to be several hundred $\mu \mathrm{m}$ order, where thousands of capillary emitters exist.

In addition to increasing the emitter array density, the sub- $\mu \mathrm{m}$ order diameter of the emitter capillary can increase the fluidic impedance for suppressing droplets emission, where the extraction of mixed ion/droplet significantly reduces the thruster efficiency. ${ }^{14)}$ The fluidic impedance is given by Eq. (1) with the assumption of the Hagen-Poiseuille flow in the capillary,

$$
\frac{128 \mu L}{\pi D^{4}},
$$

where $\mu$ is the viscosity of the fluid, $L$ is the length of the capillary, and $D$ is the diameter of the capillary. Since the fluidic impedance is inversely proportional to $D^{4}$, a small diameter capillary significantly increases the fluidic impedance. However, by a conventional MEMS etching 
method, it is difficult to fabricate a sub- $\mu \mathrm{m}$ scale capillary with a very high aspect ratio because the high-aspect-ratio etching tends to stop in the intermediate of the capillary. To solve this issue, we have developed a method to fabricate tiny cones as a base structure of the capillary emitters, which is originally based on the fabrication technique of FEAs. We have modified the above process by removing the tiny cones (as will be detailed later in Sect. 3).

The gap between the emitter and the extractor electrodes is in the range of $0.5-1 \mu \mathrm{m}$, which is approximately one-hundredth of the electrode gap of conventional electrospray thrusters. Thus, the starting voltage of ion emission, which is mainly determined by the curvature radius of the emitter tip and the distance between the emitter and the extractor, is expected to be much smaller. ${ }^{21)}$ This low-voltage operation seems to be advantageous from the viewpoint of power consumption. However, low-voltage driving also results in a low thrust and specific impulse as the thrust $T$ and the specific impulse $I_{\text {sp }}$ are respectively defined as ${ }^{13)}$

$$
\begin{gathered}
T=\sqrt{\frac{2 M}{q}} I_{\mathrm{b}} \sqrt{V_{\mathrm{b}}}, \\
I_{\mathrm{sp}}=\frac{T}{\dot{m} g_{0}}
\end{gathered}
$$

where $M$ is the ion mass, $q$ is the charge, $I_{\mathrm{b}}$ is the ion beam current, $V_{\mathrm{b}}$ is the net voltage through which the ion is accelerated, $\dot{m}$ is the mass flow rate of the propellant, and $g_{0}$ is the acceleration of gravity. It is impossible to operate our device at a higher voltage (a few $\mathrm{kV}$ ) because the breakdown between the electrodes will occur. Thus, we plan to provide an accelerator electrode separately to complement both thrust and specific impulse. The gap between the accelerator and the extractor is a few hundred $\mu \mathrm{m}$, the scale of which is very similar 
to that of the electrodes employed for ion thrusters. ${ }^{13)}$ Since there is an optimum voltage (electric field) range for ion beam extraction, ${ }^{21)}$ such separation of ion beam extraction and acceleration can be useful for the thrust control.

\section{Fabrication}

The fabrication process is mainly divided into two procedures: the top side of the double electrodes (emitter and extractor) and the bottom side of the reservoir. In several steps of the fabrication (photolithography and deep-RIE (reactive ion etching)), we used a minimal-fab system, ${ }^{24,25)}$ which consists of a group of small semiconductor manufacturing equipment for a half-inch wafer eliminating a need of cleanroom environment. The minimal-fab system is suitable for multi-product and low-volume manufacturing, and thus the system would be the best choice for the fabrication of micro space propulsion.

\subsection{Top double electrodes}

As mentioned in Sect. 2, the top double electrodes consist of integrated capillary emitters and extractor electrodes. The fabrication of the top electrodes is based on that of a volcanostructured double-gate Spindt-type FEA (VDGS-FEA), which was initially developed for image sensor applications. ${ }^{22)}$ The main feature of the VDGS-FEA fabrication is a self-aligned process for making double-layered volcano-structured electrodes, the details of which were reported elsewhere. ${ }^{22,23)}$ The center of the double-layered electrode apertures is automatically 
aligned with the same position without photolithography. Therefore, alignment with the precision of nm-scale becomes possible, which cannot be realized using wafer-level bonding of the electrodes for conventional electrospray thrusters, ${ }^{14)}$ which is a significant advantage in the fabrication of integrated electrodes.

Figure 3 shows the fabrication procedure of the top electrodes. The difference from the FEA fabrication is the Al layer deposited in the first step (Fig. 3(a)). This layer has a role in stopping deep-RIE conducted in the reservoir process. After that, Ni cones were formed on the stopping layer, where the bottom diameter of Ni cones were several hundred nanometers (Fig. $3(b)) .{ }^{22)}$ In our process, the Ni cones were only for fabricating capillary structures around them, which were removed in the fabrication procedure of the back-side reservoir and capillaries. After the formation of the cones, an insulator $\left(\mathrm{SiO}_{2}\right)$ layer and metal $(\mathrm{Nb})$ electrode were sequentially deposited to form volcano-structured electrodes. The top of the mountain structured metal electrode was selectively opened using an etch-back procedure. ${ }^{22,23)}$ The diameter of the electrode aperture, which affects ion emission characteristics as described in Sect. 2, can be precisely controlled by the etch-back process; therefore, we can easily obtain sub- $\mu \mathrm{m}$ aperture without lithography process. By applying these steps twice, we can fabricate the double layered volcano-structured electrode, as shown in Fig. 3(c).

\subsection{Back-side reservoir and capillaries of ionic liquid}

After the formation of the top double electrodes, the back-side reservoir and capillaries of ionic 
liquid were fabricated as shown in Fig. 4. Firstly, we protected the top electrodes with photoresist having a thickness of approximately $3 \mu \mathrm{m}$ (Shin-Etsu Chemical, SIPR-9740-3.0), and then the pattern of the reservoir with $300 \mu \mathrm{m}$ in diameter was formed with Al (Fig. 4(a)). Then, the Si wafer was etched from the back side to the Al stopping layer by a minimal deepRIE machine, where a 100-MHz RF generator was used to maintain inductively coupled plasma discharges for the etching. The Bosch process using $\mathrm{SF}_{6}$ and $\mathrm{C}_{4} \mathrm{~F}_{8}$ gases was conducted to penetrate the Si wafer (Fig. 4(b)), the details of which are written in Refs. 26-28. After this step, the carbon-related protective layer was considered to remain on the Al etch-stop layer and inhibit the subsequent metal etching process. To remove the layer, we conducted the plasma ashing with $\mathrm{O}_{2}$ and a small amount of fluorocarbon gas $\left(\mathrm{CHF}_{3}\right)$, which can significantly enhance the ashing rate. ${ }^{29)}$ After the formation of the reservoir and plasma ashing, the Al stopping layer and $\mathrm{Ni}$ cones were etched with dilute nitric acid to connect the reservoir and capillary emitters (Fig. 4(c)). Finally, the photoresist on the electrodes was removed using an organic solvent (Fig. $4(d))$.

Figure 5 shows scanning electron microscope (SEM) images of the $5 \mu \mathrm{m}$-pitch emitter array and an enlarged view of the emitter seen from the top side of the electrodes. As shown in the figure, $\mathrm{Ni}$ cones at the center of the electrodes were removed, and the capillary emitter structure was able to be fabricated. Figure 6(a) shows the optical microscope image of the emitter array taken from the back side after the above steps. The black dots appear in $5 \mu \mathrm{m}$ pitch, which represents the emitter array. Figure 6(b) shows a magnified SEM image of one of 
the black dots shown in Fig. 6(a), where an approximately 700-nm diameter of the capillary entrance with a through hole can be seen. Since the diameter was equal to that of the bottom of Ni cones fabricated in Fig. 3(b), we confirmed that the Al stopping layer and the Ni cones were removed successfully and ionic liquid could be supplied from the back side of the emitter array to its tips.

\section{Ion emission experiment}

\subsection{Experimental setup}

Figure 7 shows a schematic of the experimental setup for the current measurement of ion emission. In the measurement, we used an emitter array with approximately 2900 emitters in a reservoir of $300-\mu \mathrm{m}$ diameter and an ionic liquid of 1-ethyl-3-methylimidazolium tetrafluoroborate $(\mathrm{EMI}-\mathrm{BF} 4)$ or 1-ethyl-3-methylimidazolium dicyanamide (EMI-DCA) as the propellant. Since a feeding system of ionic liquid was not developed in this study, a drop of ionic liquid $(0.1 \mu \mathrm{L})$ was just put on the reservoir (the back side of the emitter array) to be supplied to the emitter array. To collect emitted ions, a Si plate deposited with Al was placed at $8.5 \mathrm{~mm}$ downstream of the emitter array as the collector. The emitter was biased by a source meter (Keithley 2657A) through a shunt resistor of $1 \mathrm{M} \Omega$. We applied 5-Hz bipolar pulse voltages to the emitter chip, the amplitude of which increased by $1 \mathrm{~V}$ at each cycle from $0 \mathrm{~V}$ to $200 \mathrm{~V}$. The extractor and the collector were also connected to another source meter (Keithley 2636A) through $100 \mathrm{k} \Omega$ and $1 \mathrm{M} \Omega$ resistors, respectively. The voltage output to the extractor 
was set at $0 \mathrm{~V}$ (equivalent to the ground potential), which could detect the ions colliding against the extractor. The collector was biased by $5-\mathrm{Hz}$ bipolar pulse voltage of $100-\mathrm{V}$ amplitude and its polarity was always opposite to that of the emitter voltage. Note that owing to the highfrequency harmonics, the collector current drastically increased or decreased for the first $10 \mathrm{~ms}$ at the time of voltage alternation, showing the capacitor characteristics; therefore, we discarded the data for the first $10 \mathrm{~ms}$ and obtained the collector current averaging the rest of data for 90 ms. The experiment was conducted in a vacuum chamber where the pressure was maintained below $1.0 \times 10^{-3} \mathrm{~Pa}$.

\subsection{Experimental results and discussion}

Firstly, we conducted the ion emission experiment of EMI-BF4, which was often utilized in previous studies of electrospray thrusters, ${ }^{14,17,19,21)}$ but almost no collector current was observed. Figure 8 shows the SEM image of the high-density emitter array after the experiment, where the ionic liquid was removed by acetone before the observation. Some of the $\mathrm{Nb}$ double electrodes seemed to disappear probably by electrochemical reaction with $\mathrm{EMI}^{-\mathrm{BF}}$ 4. Therefore, we describe the experimental results utilizing EMI-DCA hereafter, which would not cause electrochemical reaction with the $\mathrm{Nb}$ electrodes because it does not include fluorine in its anion.

Figure 9 shows the measured currents of the emitter, extractor, and collector as functions of emitter voltage for its amplitude range from 80 to $92 \mathrm{~V}$. When the emitter voltage was lower than $-87 \mathrm{~V}$ or higher than $89 \mathrm{~V}$, the collector current began to be detected and 
reached $-1.0 \mu \mathrm{A}$ at $-92 \mathrm{~V}$ and $0.6 \mu \mathrm{A}$ at $92 \mathrm{~V}$. Since the starting voltage of ion extraction is a function of the surface tension of the ionic liquid, the curvature radius of the emitter tip, and the distance between the emitters and the extractor, ${ }^{30)}$ the absolute value of the starting voltage is estimated to be approximately equal for both cation and anion extraction, which was true for the present experiments and the previous ones. ${ }^{17,21,31)}$ We could demonstrate that ions were extracted from the sub- $\mu \mathrm{m}$ scale capillaries of the high-density emitter array when EMI-DCA was utilized as the propellant. Note that the sum of the extractor current and the collector current was approximately equal to the emitter current, indicating the current conservation via emitted ions, and thus the plots of the emitter current at the voltage amplitude of $92 \mathrm{~V}$ were out of range of the graph.

Figure 9 also indicates that the emitter and the extractor currents were nonnegligible values and almost the same before the ion emission. Since no emitter and extractor currents were observed without ionic liquid, this phenomenon might be due to the slight leakage of the ionic liquid from the capillary emitters into the space between the emitter and the extractor although the resistance value of the ionic liquid was not constant (e.g., approximately from 130 to $150 \mathrm{M} \Omega$ at the voltage in the range $80-89 \mathrm{~V}$ for almost constant current of $0.6 \mu \mathrm{A}$ ). When the emitter voltage was higher or lower than $\pm 92 \mathrm{~V}$, the emitter and the extractor currents increased or decreased suddenly, exceeding $\pm 50 \mu \mathrm{A}$, and the ion emission was hindered. Figure 10 shows the SEM image of the emitter array after a voltage of up to $200 \mathrm{~V}$ was applied to the emitter. One of the $\mathrm{Nb}$ double electrodes clearly disappeared and the leaked ionic liquid seemed 
to be solidified on the surface, which was not observed before the voltage was applied, implying that the emitter and extractor were broken due to the electrical short circuit of between the two electrodes through the leaked ionic liquid at voltage amplitudes of $>92 \mathrm{~V}$.

Finally, we compare the current density of the present high-density emitter array with that of conventional electrospray thrusters. As shown in Fig. 9, the current density was determined to be $-1.4 \mathrm{~mA} / \mathrm{cm}^{2}$ at an emitter voltage of $-92 \mathrm{~V}$ and $0.85 \mathrm{~mA} / \mathrm{cm}^{2}$ at $92 \mathrm{~V}$, where the emission area was assumed to be equal to the reservoir area (300 $\mu \mathrm{m}$ in diameter). As a typical example of the conventional electrospray thrusters, a scalable ion electrospray propulsion system (S-iEPS), which had a porous emitter array of 480 emitter tips in the emission area of $9 \times 9 \mathrm{~mm}^{2}$, produced the current of $-250 \mu \mathrm{A}$ at the emitter voltage of $-900 \mathrm{~V}$ and 200 $\mu \mathrm{A}$ at $900 \mathrm{~V}$ with the same ionic liquid of EMI-DCA, ${ }^{17,31)}$ resulting in the current density of $-0.31 \mathrm{~mA} / \mathrm{cm}^{2}$ at $-900 \mathrm{~V}$ and $0.25 \mathrm{~mA} / \mathrm{cm}^{2}$ at $900 \mathrm{~V}$. In comparison, our high-density emitter array obtained a few times higher current density for both polarities than the S-iEPS and showed a similar tendency that the anion produced higher absolute value of the current than the cation at the same absolute value of the emitter voltage owing to the lighter mass of DCA $(66 \mathrm{~g} / \mathrm{mol})$ than EMI $(111 \mathrm{~g} / \mathrm{mol})$. Moreover, since the thrust is proportional to the current and the square root of the beam voltage from Eq. (2), our high-density emitter array is expected to deliver at least 10 times higher thrust density when the accelerator electrode is placed and the potential difference between the emitter and the accelerator is set at $900 \mathrm{~V}$. Although the magnitude of the collector current was still lower than that of the extractor current and the voltage range that 
could extract ions was narrow probably owing to the leakage of the ionic liquid as shown in Figs. 9 and 10, these issues are expected to be solved when an appropriate feed mechanism of the ionic liquid is developed. As mentioned in Sect. 4.1, the ionic liquid was just placed on the back side of the emitter array and its flow control was not taken, and thus, when the voltage was applied, the whole ionic liquid probably moved and leaked out. If the resistance force can be supplied to the ionic liquid in the opposite direction to the ion emission, the leakage might be suppressed, which would contribute to the increase in ion current.

\section{Conclusions}

In this paper, we have proposed and fabricated a high-density emitter array for electrospray thrusters and conducted current measurements as a preliminary experiment for the demonstration of the thruster operation. To realize high-density packaging, instead of MEMS etching techniques, we have utilized the fabrication technique for FEAs. Since sub- $\mu$ m scale of capillary electrodes can be fabricated, the emitters pitch is reduced to $5 \mu \mathrm{m}$, and thus the emitter density of about 4 million $/ \mathrm{cm}^{2}$ is realized, which is four orders of magnitude higher than that of conventional electrospray thrusters. After the same structure as an FEA was fabricated on the Si wafer except thin Al stopping layer, deep-RIE and the etching of $\mathrm{Ni}$ cones and $\mathrm{Al}$ stopping layer were conducted to fabricate capillary emitters with self-aligned extractor electrodes and reservoirs for the propellant of the ionic liquid. As a result, we have demonstrated that the sub- $\mu \mathrm{m}$ scale capillary emitters could be fabricated successfully. For the 
current measurements, EMI-BF4 and EMI-DCA were employed as the propellant. Since EMI$\mathrm{BF}_{4}$ includes fluorine, the emitter and extractor electrodes made of $\mathrm{Nb}$ underwent electrochemical reaction with the ionic liquid and failed to extract ion beams. However, the collector currents due to the ion emission from both cations and anions were detected at emitter voltages of approximately $\pm 90 \mathrm{~V}$ when EMI-DCA, which does not include fluorine, was used for the current measurement. We have also demonstrated that ions were able to be extracted from the sub- $\mu \mathrm{m}$ scale capillaries of the high-density emitter array. Although the absolute value of the detected current was $1.0 \mu \mathrm{A}$ at the maximum probably owing to the leakage of the ionic liquid, the current density was nonetheless a few times higher than that of the conventional electrospray thrusters using ionic liquids. Since the applied voltage was one-tenth of that of the conventional thruster, the thrust density should be at least one order of magnitude higher when the accelerator electrode is employed for producing the same beam voltage of $900 \mathrm{~V}$. The leakage of ionic liquids would also be avoided if a propellant feed mechanism is properly developed, which is left as our future work.

\section{Acknowledgment}

This work was supported in part by JSPS KAKENHI Grant Number JP18H01623, the Asahi Glass Foundation, SEI Group CSR Foundation, Yokohamakogyokai Research Aid Foundation, and JAXA/RDD. 


\section{References}

1) H. Heidt, J. Puig-Suari, A. S. Moore, S. Nakasuka, and R. J. Twiggs, 14th the AIAA/USU Conf. Small Satellites, 2000, SSC00-V-6.

2) M. Taraba, C. Rayburn, A. Tsuda, and C. MacGillivray, 23th the AIAA/USU Conf. Small Satellites, 2010, SSC09-X-6.

3) D. C. Alhorn, J. P. Casas, E. F. Agasid, C. L. Adams, G. Laue, C. Kitts, and S. O'brien, 25th the AIAA/USU Conf. Small Satellites, 2011, SSC11-VI-1.

4) S. Kim, T. Eishima, N. Miyashita, Y. Nojiri, and Y. Nakamura, 24th the AIAA/USU Conf. Small Satellites, 2010, SSC10-II-6.

5) W. Blackweii, G. Allen, C. Galbraith, T. Hancock, R. Lestile, I. Osaretin, L. Retherford, M. Scartio, C. Semisch, M. Shields, M. Silver, D. Toher, K. Wight, D. Miller, K. Cahoy, and N. Erickson, 12th Specialist Meeting on Microwave Radiometry and Remote Sensing of the Environment (MicroRad), 2012, 12691701.

6) T. Iso, K. Takahashi, T. Hiramoto, and M. Kawamura, Joint Conf. 31th Int. Symp. Space Technology and Science, 26th Int. Symp. on Space dynamics, and 8th Nano-satellite Symp., 2017, ISTS-2017-f-081.

7) H. Koizumi, K. Komurasaki, J. Aoyama, and K. Yamaguchi, Trans. Jpn. Soc. Aeronaut. Space. Sci., Aerosp. Technol. Jpn. 12, Tb_19(2014).

8) H. Koizumi, H. Kawahara, K. Yaginuma, J. Asakawa, Y. Nakagawa, Y. Nakamura, S. Kojima, T. Matsuguma, R. Funase, J. Nakatsuka, and K. Komurasaki, Trans. Jpn. Soc. Aeronaut. Space. Sci., Aerosp. Technol. Jpn. 14, Pb_13 (2016).

9) J. Asakawa, H. Koizumi, K. Nishii, N. Takeda, M. Murohara, R. Funase, and K. Komurasaki, Trans. Jpn. Soc. Aeronaut. Space. Sci., Aerosp. Technol. Jpn. 16, 427 (2018).

10) SpaceWorks, 2018 Nano/Microsatellites Marcket Forecast, 2018.

11) S. J. Chung, S. Bandyopadhyay, R. Foust, G. P. Subramanian, and F. Y. Hadaegh, J. Spacecr. Rockets, 53, 567 (2016).

12) M. M. Micci and A. D. Ketsdever, Micropropulsion for Small Spacecraft (American Institute of Aeronautics and Astronautics, Reston, VA, 2000).

13) D. M. Goebel and I. Katz, Fundamentals of Electric Propulsion; Ion and Hall Thrusters (Wiley, Hoboken, NJ, 
2008).

14) S. Dandavino, C. Ataman, C. N. Ryan, S. Chakraborty, D. Courtney, J. P. W. Stark, and H. Shea, J. Micromech. Microeng. 24, 075011 (2014).

15) G. Taylor, Proc. R. Soc. London, Ser. A 280, 383 (1964).

16) H.Watanabe, Hyomen Kagaku 28, 298 (2007) [in Japanese].

17) D. Krejci, F. Mier-Hicks, R. Thomas, T, Haag, and P. Lozano, J. Spacecr. Rockets 54, 447 (2017).

18) E. Grustan-Gutierrez and M. Gamero-Castaño, J. Propul. Power 33, 984 (2017).

19) D. G. Courtney, S. Dandavino, and H. Shea, J. Propul. Power 32, 392 (2016).

20) R. Krpoun and H. R. Shea, J. Micromech. Microeng. 19, 0450919 (2009).

21) K. Nakagawa, T. Tsuchiya, and Y. Takao, Jpn. J. Appl. Phys. 56, 06GN18 (2017).

22) M. Nagao, Y. Gotoh, Y. Neo, and H. Mimura, J. Vac. Sci. Technol. B 13, 02 G108 (2016).

23) M. Nagao and T. Yoshida, Microelectron. Eng. 132, 14 (2015).

24) S. Khumpuang and S. Hara, IEEE Trans. Semicond. Manuf. 28, 393 (2015).

25) S. Khumpuang, F. Imura, and S. Hara, IEEE Trans. Semicond. Manuf. 28, 551 (2015).

26) F. Laermer and A. Schilp, U.S. Patent 5501893 (1996).

27) B. Wu, A. Kumar, and S. Pamarth, J. Appl. Phys. 108, 051101 (2010).

28) Y. Liu, H. Tanaka, N. Umeyama, K. Koga, S. Khumpuang, M. Nagao, T. Matsukawa, and S. Hara, Jpn. J. Appl. Phys. 57, 06 HD03 (2018).

29) J. J. Hannon and J. M. Cook, J. Electrochem. Soc. 131, 1164 (1984).

30) P. Lozano, M. Martínez-Sánchez, and J. M. Lopez-Urdiales, J. Colloid Interface Sci. 276, 392 (2004).

31) C. Guerra-Garcia, D. Krejci, and P. Lozano, J. Phys. D 49, 115503 (2016). 


\section{List of Figure Captions}

Fig. 1. (Color online) Schematic of an internally fed electrospray thrusters. High voltage bipolar pulse is applied to the emitter electrode while the extractor electrode is grounded. High positive/negative voltage extracts positive/negative ions (cations/anions) from the ionic liquid.

Fig. 2. (Color online) Schematic of a high-density emitter array together with an extractor and accelerator electrodes. Sub- $\mu \mathrm{m}$ scale capillary emitters with $5 \mu \mathrm{m}$ pitch can be fabricated within several hundred $\mu \mathrm{m}$ diameters of the ionic liquid reservoir. The density of the emitter array can reach approximately 4 million $/ \mathrm{cm}^{2}$, which is four orders of magnitude higher than that of conventional electrospray thrusters.

Fig. 3. (Color online) Fabrication procedure of the top electrodes. (a) Firstly, the thin Al layer was deposited on the Si wafer, which has the role of stopping deep-RIE performed in the reservoir process. (b) Then, Ni cones were formed on the Al layer. (c) After that, double electrodes were fabricated by the etch-back technique.

Fig. 4. (Color online) Fabrication procedure of the reservoir structure. (a) Firstly, we protected the top electrodes with thick photoresist, and the pattern of the reservoir was formed with Al. (b) Then, deep-RIE was conducted to penetrate the Si wafer. (c) After that, the Al stopping 
layer and Ni cones were etched with dilute nitric acid. (d) Finally, the photoresist on the electrodes was removed using an organic solvent.

Fig. 5. SEM images of (a) the $5 \mu \mathrm{m}$-pitch emitter array and (b) an enlarged SEM view of the emitter seen from the top of the electrodes.

Fig. 6. (Color online) (a) Optical microscope image of the emitter array and (b) magnified SEM image of a single capillary, where both images were taken from the back side of the emitter array. A capillary having several hundred $\mathrm{nm}$ diameters at the entrance was found to be fabricated.

Fig. 7. (Color online) Schematic of the experimental setup for the current measurement of the ion emission.

Fig. 8. SEM image of the emitter array after the experiment utilizing EMI-BF4 as the propellant. Some of the $\mathrm{Nb}$ double electrodes disappeared owing to the electrochemical reaction with EMI$\mathrm{BF}_{4}$.

Fig. 9. (Color online) Collector current (solid circles), extractor current (open squares), and 
emitter current (open triangles) as functions of the emitter voltage for the emission of (a) anions and (b) cations of EMI-DCA. Note that the plots are shown only for the voltage range (a) from -92 to $-80 \mathrm{~V}$ and (b) from 80 to $92 \mathrm{~V}$.

Fig. 10. SEM image of the emitter array after the experiment of Fig. 9, where up to $\pm 200 \mathrm{~V}$ of the emitter voltage was applied. One of the $\mathrm{Nb}$ double electrodes disappeared and the leaked ionic liquid seemed to be solidified on the surface. 


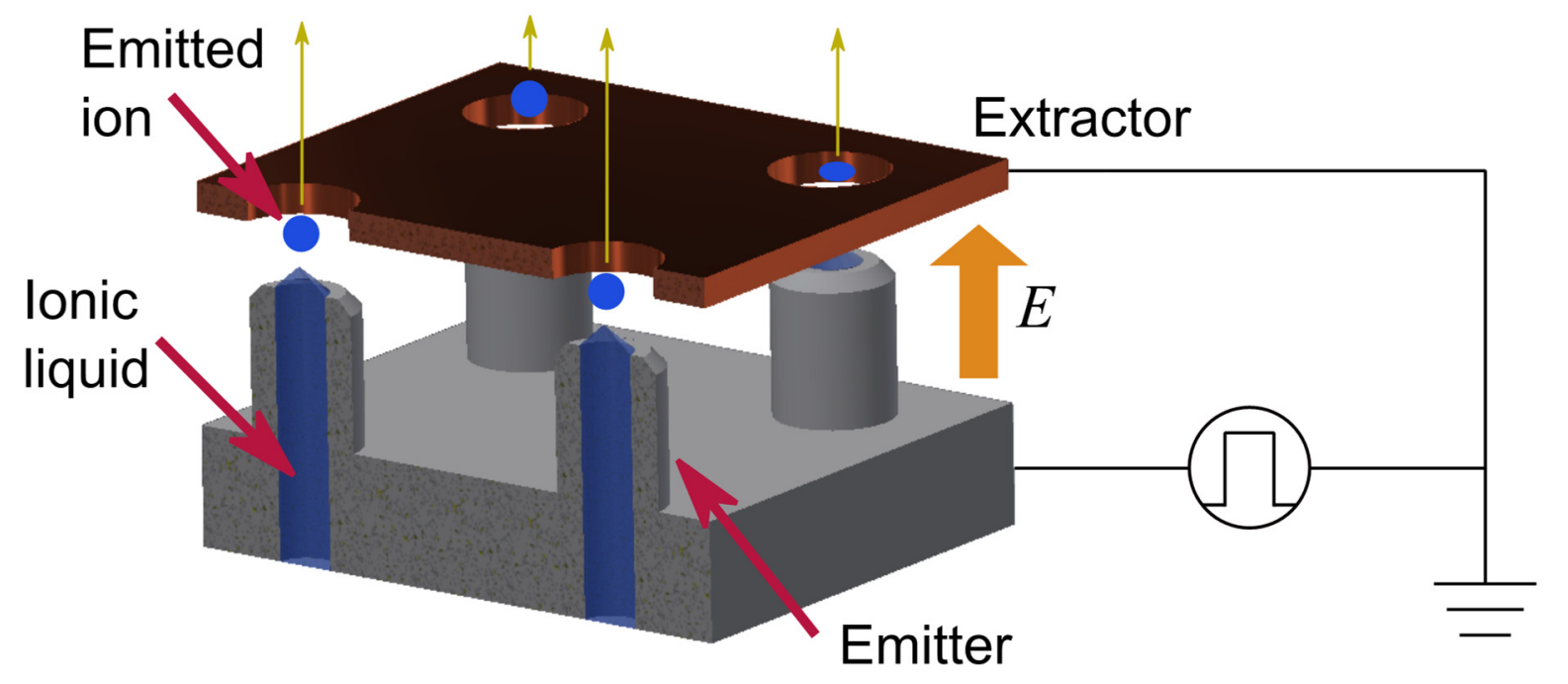

Fig. 1 


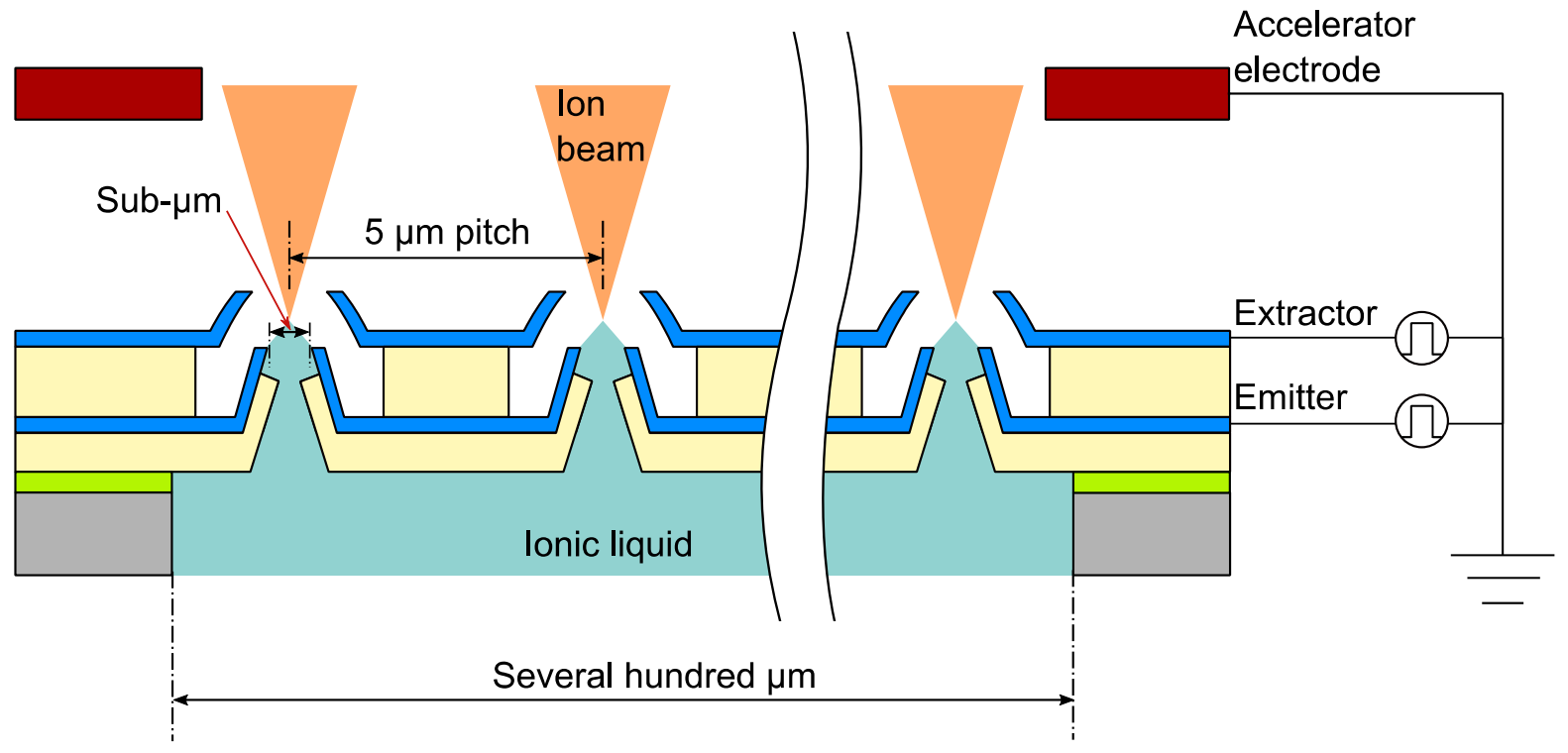

Fig. 2 
(a) Deposition of Al stopping layer

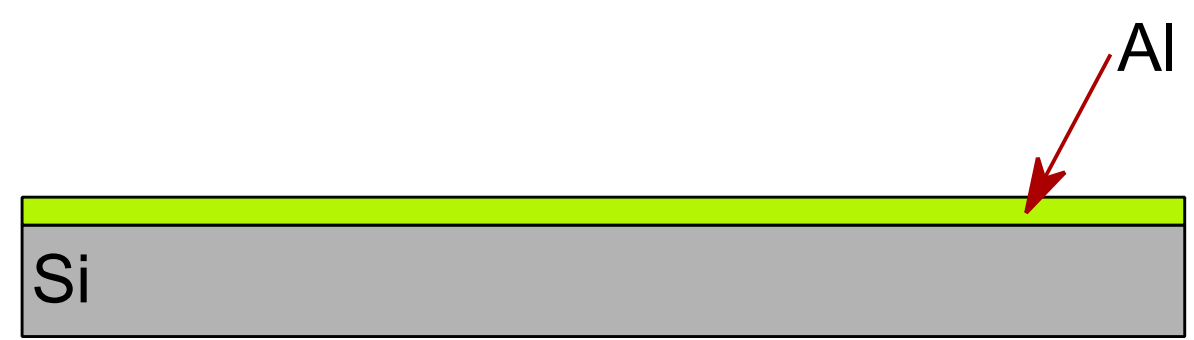

(b) Formation of Ni cones
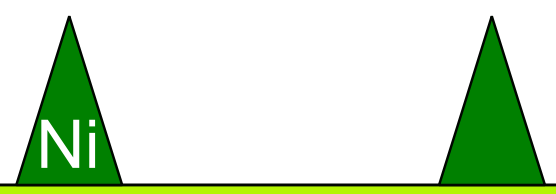

(c) Construction of double electrodes by etch-back tecnique

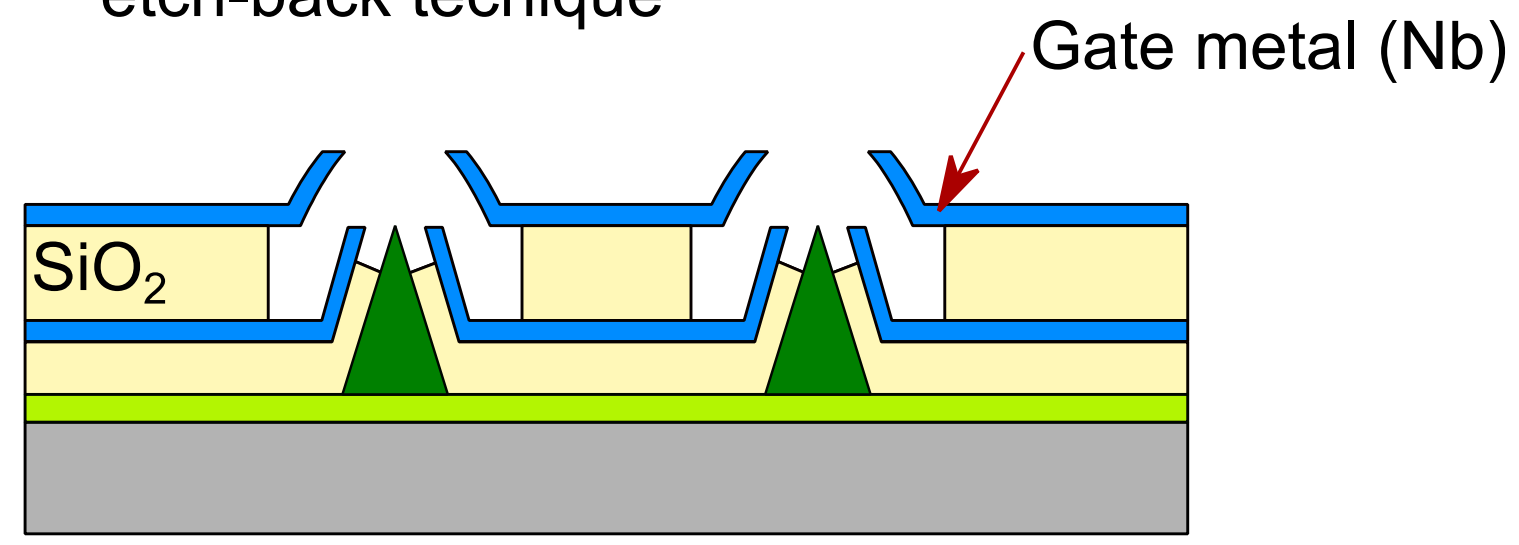

Fig. 3 
(a) Protection of top electrodes and hole patterning

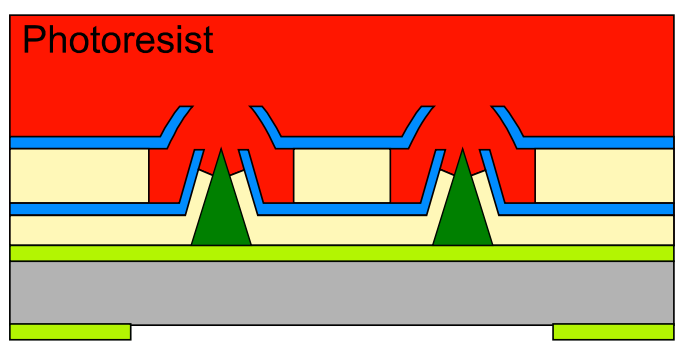

(c) Etching of Al layers and Ni emitters

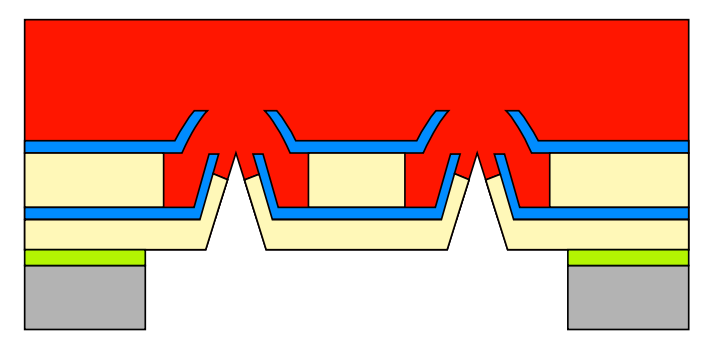

(b) Deep-RIE from backside of Si wafer

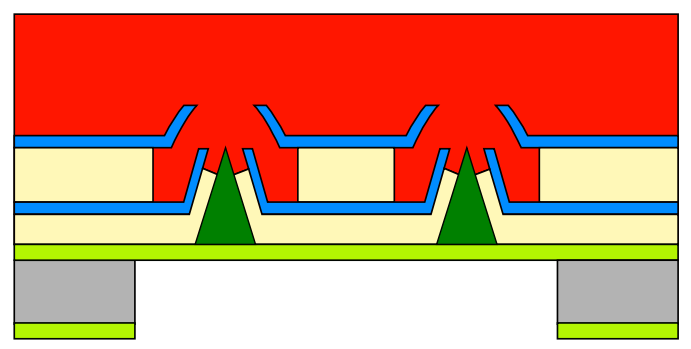

(d) Removal of photoresist

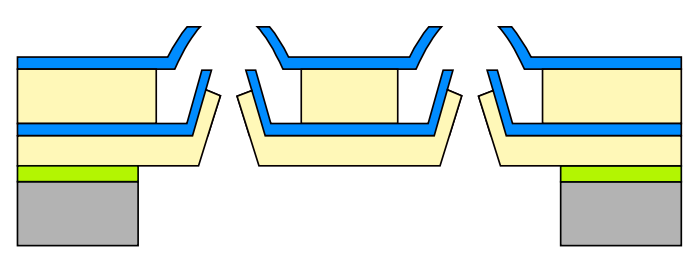

Fig. 4 

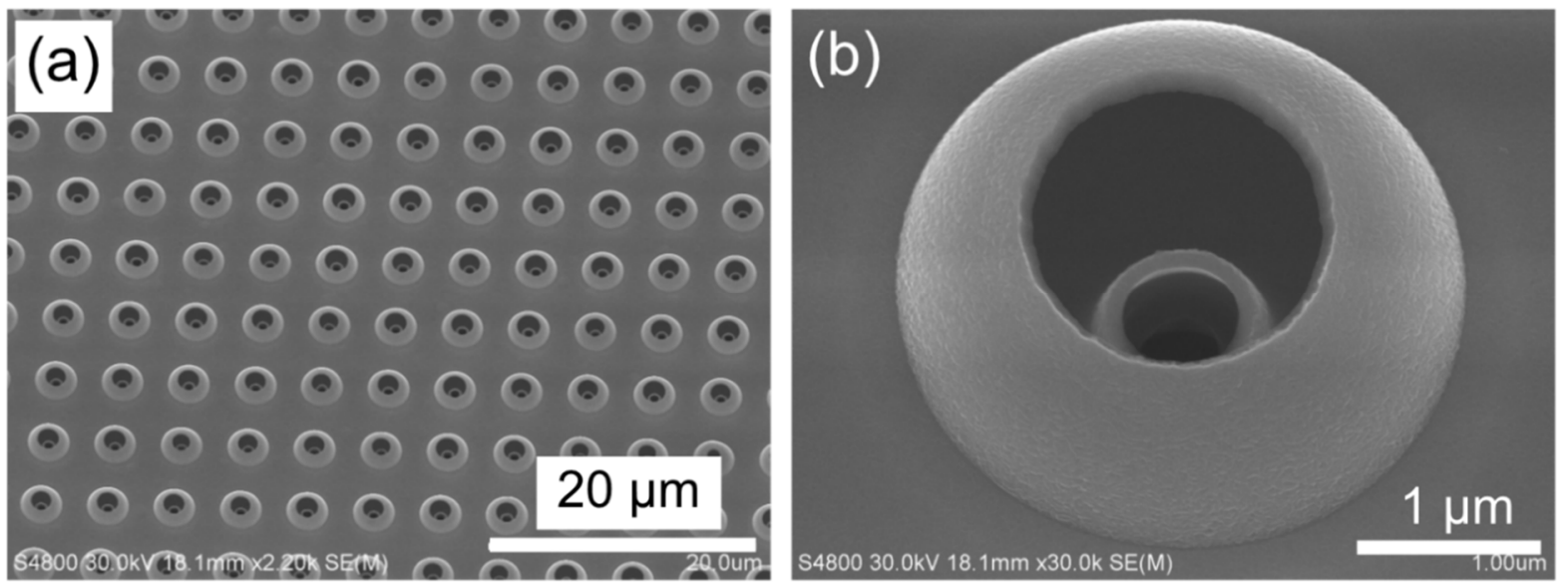

Fig. 5 


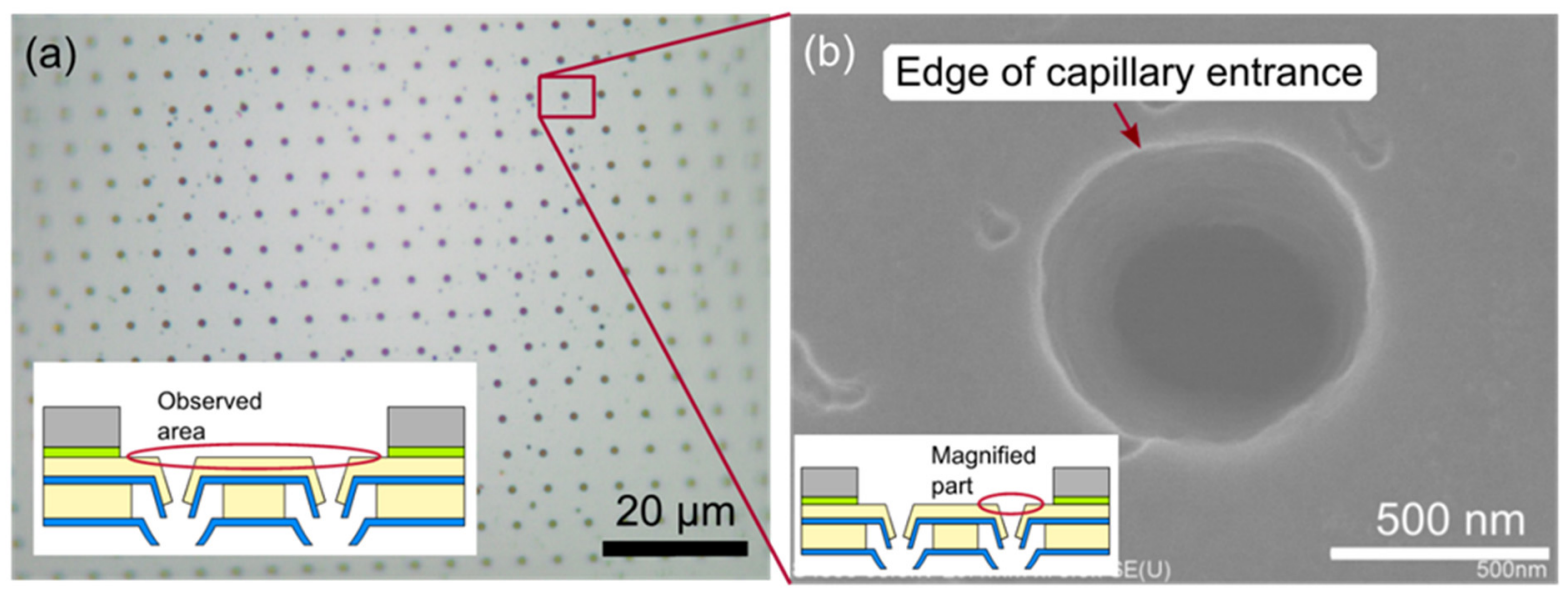

Fig. 6 


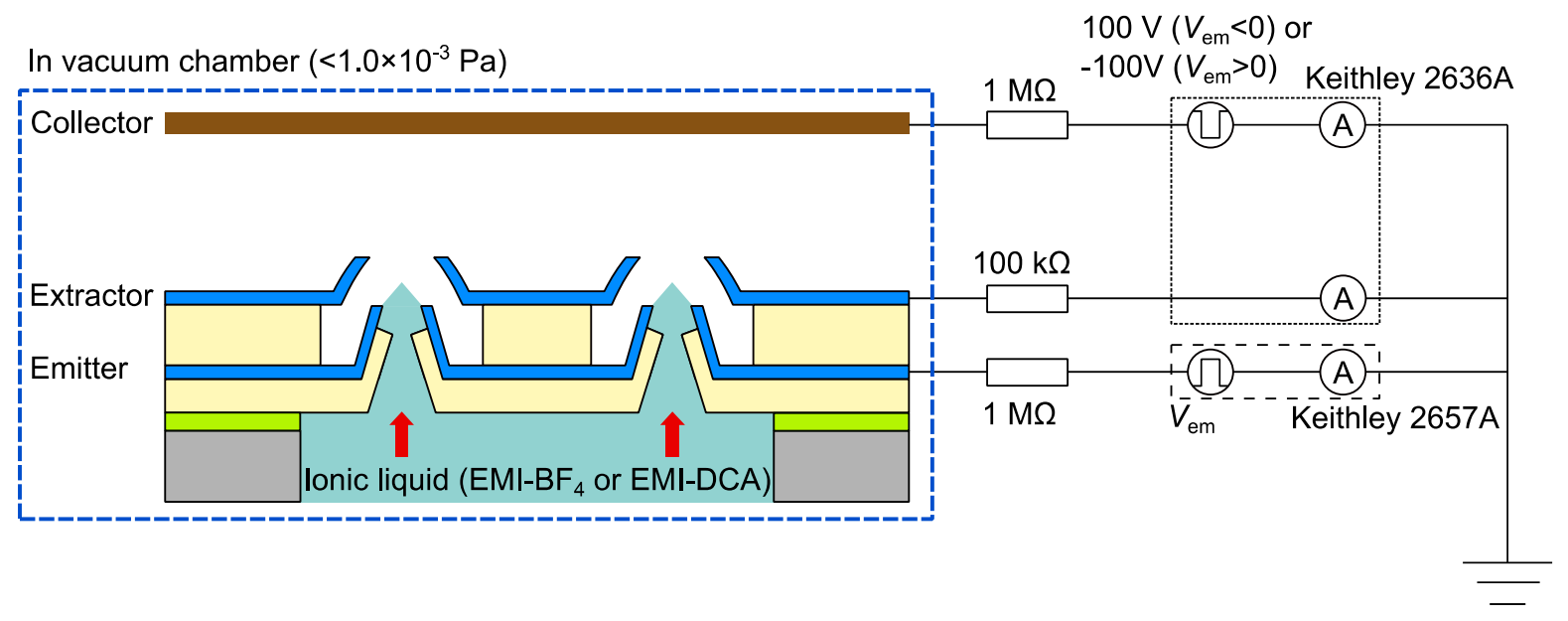

Fig. 7 


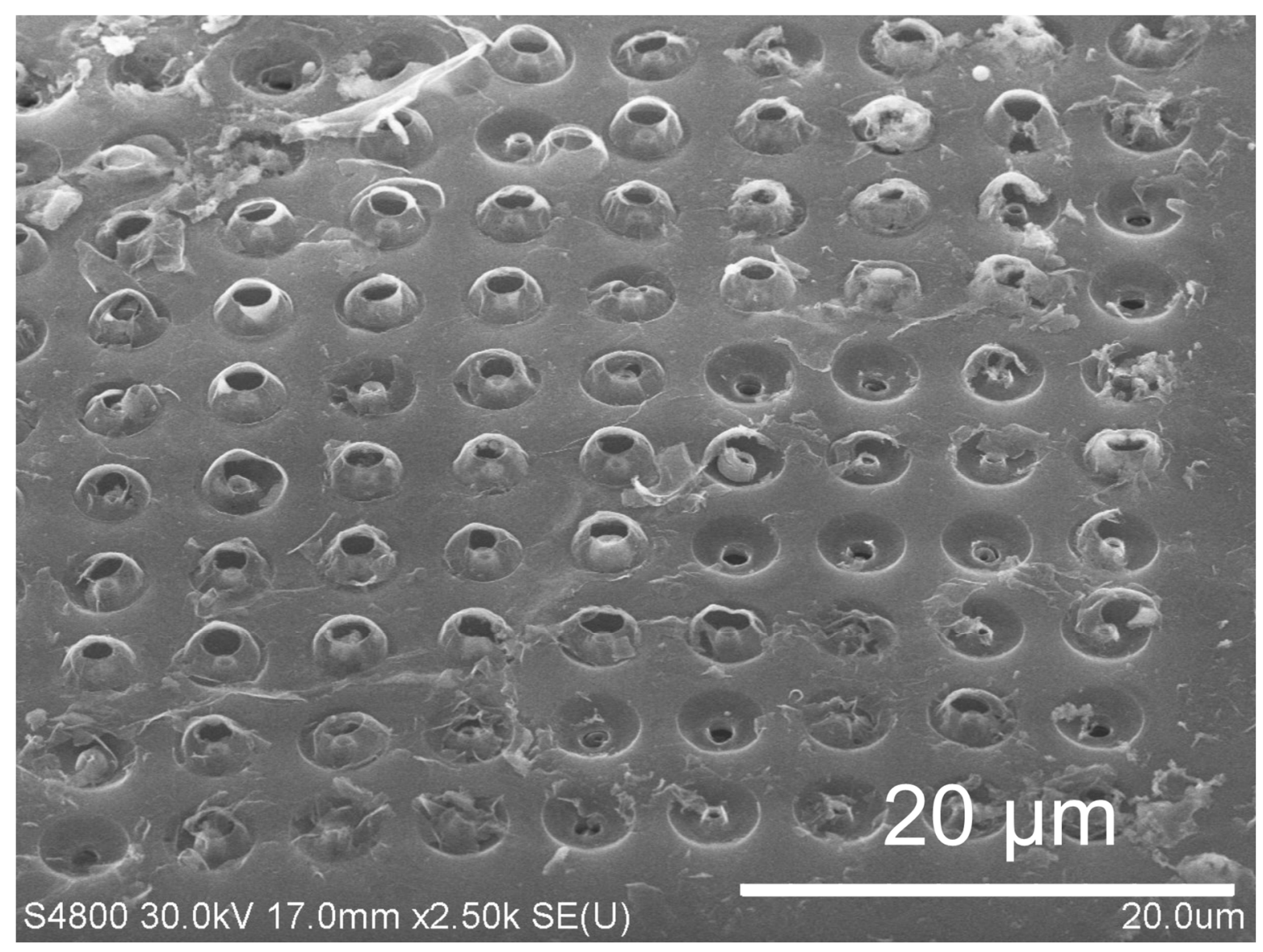

Fig. 8 
(a) Emission of anions

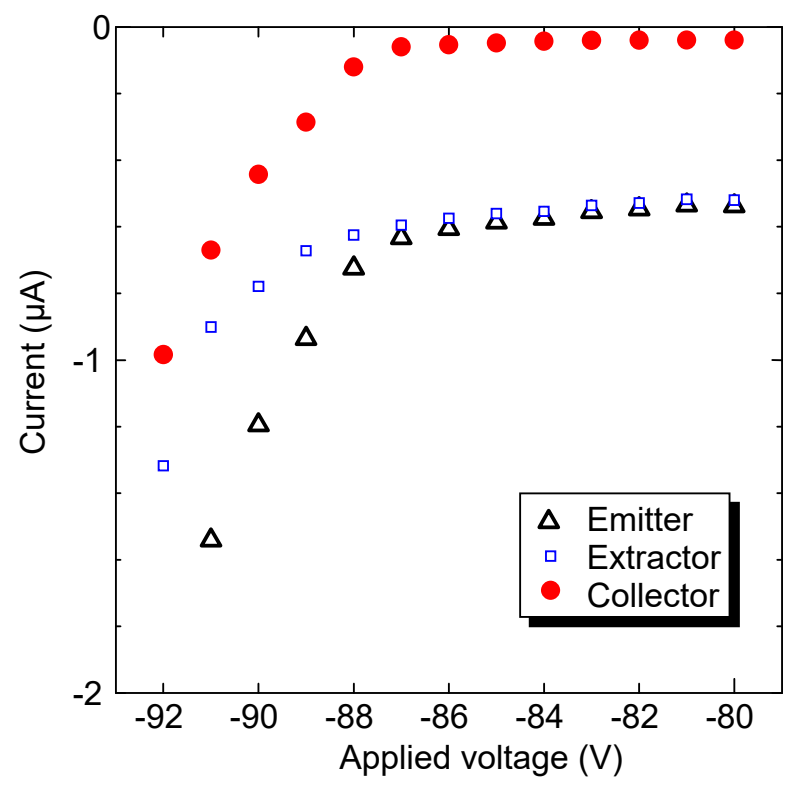

(b) Emission of cations

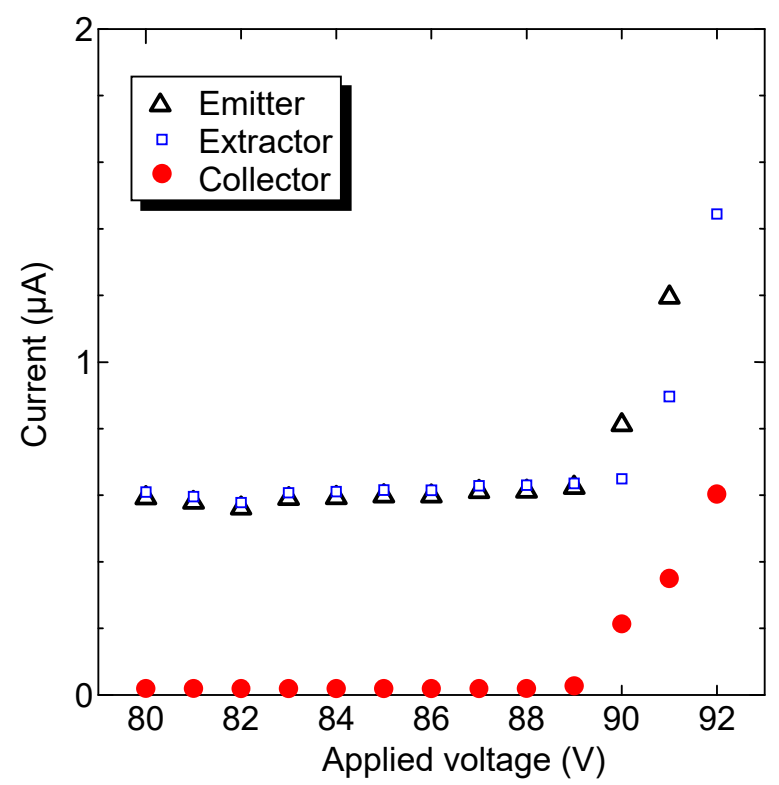

Fig. 9 


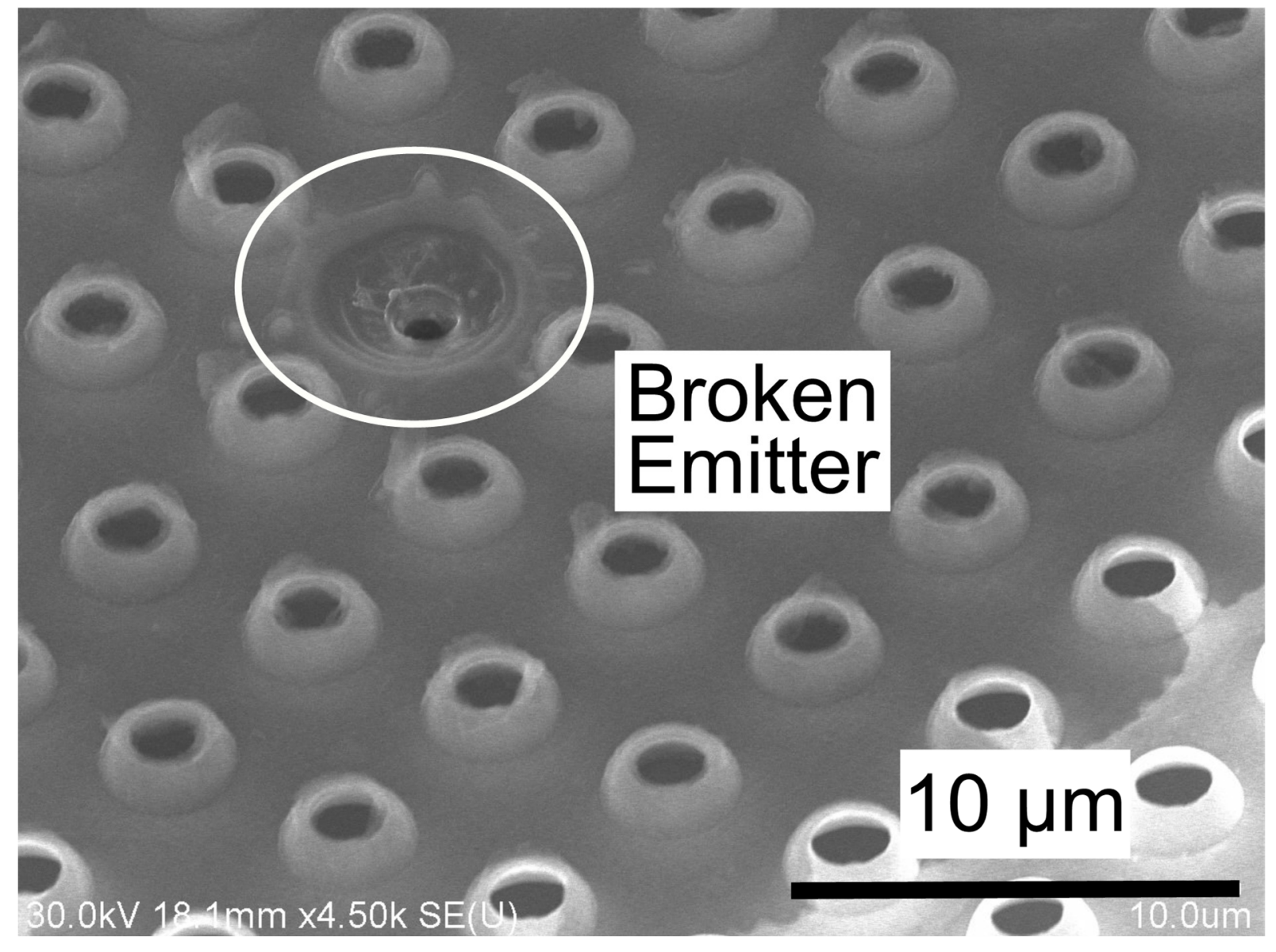

Fig.10 